\title{
Estrogen Rapidly Enhances Incisional Pain of Ovariectomized Rats Primarily through the G Protein-Coupled Estrogen Receptor
}

\author{
Guanghui An ${ }^{1}$, Wenhui $\mathrm{Li}^{2}$, Tao Yan ${ }^{1}$ and Shitong $\mathrm{Li}^{1}{ }^{1, *}$ \\ 1 Department of Anesthesiology, Shanghai First People's Hospital, Shanghai 200080, China; \\ E-Mails: gh_An@163.com (G.A.); yantao74@gmail.com (T.Y.) \\ 2 Department of Anesthesiology, Obstetrics and Gynecology Hospital of Fudan University, \\ Shanghai 200090, China; E-Mail: panhongliang2000@ gmail.com \\ * Author to whom correspondence should be addressed; E-Mail: lishitongs@ @otmail.com; \\ Tel.: +86-21-632-400-90; Fax: +86-21-640-858-75.
}

Received: 20 April 2014; in revised form: 10 May 2014 / Accepted: 13 May 2014 /

Published: 11 June 2014

\begin{abstract}
It has become increasingly apparent that the pain threshold of females and males varies in an estrogen dependent manner. To investigate the modulation of pain by estrogen and the molecular mechanisms involved in this process. A total of 48 rats were ovariectomized (OVX). At 14 and 20 days after OVX, rats were divided into eight groups: groups 1-4 were administered drugs intravenously (IV); groups 5-8 were administered through intrathecal (IT) catheter. Hind paw incision was made in all animals to determine incisional pain. Paw withdraw threshold (PWT) was tested prior to and $24 \mathrm{~h}$ after incision. The test drugs were applied $24 \mathrm{~h}$ after the incision. Rats were either IV or IT administered with: 17- $\beta$-estradiol (E2), G protein-coupled estrogen receptor (GPER)-selective agonist (G1), GPER-selective antagonist (G15) and E2 (G15 + E2), or solvent. Before and $30 \mathrm{~min}$ after IV drug administration and 20 min during the IT catheter administration, PWT was tested and recorded. $24 \mathrm{~h}$ after incisional surgery, the PWT of all rats significantly decreased. Both in the IV group and IT group: administration of E2 and G1 significantly decreased PWT. Neither administration of G15 + E2 nor solvent significantly changed PWT. Estrogen causes rapid reduction in the mechanical pain threshold of OVX rats via GPER.
\end{abstract}


Keywords: estrogen; estrogen receptors; G protein-coupled estrogen receptor (GPER); incisional pain

\section{Introduction}

It has been shown that estrogen can influence pain threshold. In a study of healthy subjects, tested by a variety of stimuli including thermal stimulation, pressure stimulation and chemical stimulation, the pain threshold and tolerance were found to be lower in females than males [1-4]. In addition, females suffered more from clinical pain disorders than males in terms of migraines and trigeminal neuralgia [5].

In animals, the basal mechanical pain thresholds of male and female rats vary in an estrogen-dependent manner [6]. Administration of estrogen has been shown to result in sensitization of nociceptive neurons, resulting in a decrease of the pain threshold [7,8]. The paw withdraw threshold (PWT) significantly reduced after subcutaneous injection of estrogen into the paw. Subcutaneous injection of the selective agonist of the G protein-coupled estrogen receptor (GPER), GPER-selective agonist (G1) had similar results [9]. In the study by Evard et al. (2004), blockade of the endogenous synthesis of estrogens in quail markedly reduced the behavioral responsiveness to painful thermal stimulus within 1-5 min, and recovered within $30 \mathrm{~min}$, indicating that estrogen had a rapid non-genomic effect on pain in the central nervous system (CNS) [10].

The results of studies investigating the effect of estrogen on pain often vary. The formalin pain model is commonly used as a model of chronic pain. Studies have shown that both estrogen replacement and administration of the estrogen receptor antagonist, tamoxifen, in the formalin model results in an anti-hyperalgesic effect [11,12]. In contrast, it has been reported that in a model of ovariectomized (OVX) rats with lower circulating levels of estrogen, the response to repetitive nociceptive stimulation was not altered compared to non-ovariectomized rats [13]. It has also been reported however, that OVX induces a hyperalgesic state of slow onset and long duration that can be reversed by estrogen [14].

Recently, the rapid effects of estrogen on nociceptive neurons have been reported. Studies have shown a reduction in PWT within a few minutes of a subcutaneous or intrathecal (IT) injection of estrogen $[9,14]$. Whilst several studies have reported that estrogen can induce a rapid increase in intracellular cAMP and $\mathrm{Ca}^{2+}$ concentration [1,15-17], the molecular mechanisms underlying the effects of estrogen remain unclear. In particular, few studies have investigated the role of the estrogen receptors in pain modulation. It is hypothesized that the reduction in pain threshold reported in conjunction with increased circulating estrogen may be modulated by estrogen receptors. The aim of the current study was to investigate the effects of estrogen in pain modulation and the possible molecular signaling pathways involved in this process.

A member of the novel protein kinase $\mathrm{C}$ family, $\mathrm{PKC} \varepsilon$, has been identified as an important intracellular mediator leading to the onset of mechanical hyperalgesia [18]. A study by Hucho et al. (2006) showed that estrogen controls PKC $\varepsilon$-dependent mechanical hyperalgesia through direct action on nociceptive neurons [9]. Kuhn et al. reported similar results whereby GPER-selective agonist (G1) and ICI 182,780, a high affinity estrogen receptor antagonist, lead to translocation of PKCE in dorsal root 
ganglion (DRG) neurons to the plasma membrane [19]. Injection of G1 or ICI 182,780 into the hind paw of male rats rapidly induced $\mathrm{PKC} \varepsilon$-dependent mechanical hyperalgesia and $\mathrm{PKC} \varepsilon$-specific inhibitory peptide, EV1-2, could completely abolish the onset of estrogen-induced mechanical hyperalgesia [19]. These studies indicate that estrogen induces quick mechanical hyperalgesia through a PKCe-dependent pathway.

The $N$-methyl-D-aspartate (NMDA) receptor (NR) plays an important role in the initiation and maintenance of nociception. Both estrogen receptor $\alpha(\mathrm{ER} \alpha)$ and the NR1 subunit of the NMDA receptor are co-expressed in dorsal horn neurons, supporting a direct action of estradiol on NMDA receptors. The possible mechanisms include increasing the expression of NMDA receptors and changing the level of subunit phosphorylation [20]. The P2X (ligand-gated non-selective cation channel P2 purinoceptors ) receptors are expressed in DRG neurons. Studies have suggested that the $\mathrm{P} 2 \mathrm{X}$ receptors in sensory neurons play a role in the generation and/or modulation of pain signaling from the periphery to the spinal cord [21]. Furthermore, it has been reported that estrogen inhibits P2X3 (ligand-gated non-selective cation channel P2 purinoceptors 3) receptor expression through a genomic mechanism [22] and the up-regulation of P2X3 receptors in DRG neurons from the OVX female rats may play a central role in mediating the abnormal nociceptive responses [23]. The mechanisms underlying the estrogen and P2X3 receptor-mediated nociceptive responses, however remain unclear.

\section{Results and Discussion}

\subsection{Hind-Paw Withdrawal Threshold (PWT)}

There was no significant difference in PWT between the groups before the incision surgery, and the PWT dropped significantly $24 \mathrm{~h}$ after the surgery (Table 1, Figure 1A-C). Still there were no significant differences between each groups $24 \mathrm{~h}$ after the surgery. The data of each group are shown in Table 1, Figure $1 \mathrm{~A}-\mathrm{C}$.

Table 1. The paw withdrawal threshold (PWT) of all the groups, before surgery, before drugs and after drugs.

\begin{tabular}{ccccc}
\hline Administration & Group & Before Surgery & Before Drugs & After Drugs \\
\hline & Solvent & $24.8 \pm 0.78 \mathrm{~g}$ & $5.23 \pm 0.72 \mathrm{~g}$ & $5.00 \pm 0.71 \mathrm{~g}$ \\
$\mathrm{IV}$ & $\mathrm{E} 2$ & $25.9 \pm 0.88 \mathrm{~g}$ & $5.03 \pm 0.74 \mathrm{~g}$ & $2.65 \pm 0.66 \mathrm{~g}$ \\
& $\mathrm{G} 1$ & $25.4 \pm 0.78 \mathrm{~g}$ & $4.87 \pm 0.40 \mathrm{~g}$ & $2.50 \pm 0.58 \mathrm{~g}$ \\
& $\mathrm{E} 2+\mathrm{G} 15$ & $24.8 \pm 0.78 \mathrm{~g}$ & $4.67 \pm 0.40 \mathrm{~g}$ & $4.60 \pm 0.51 \mathrm{~g}$ \\
& Solvent & $25.4 \pm 0.78 \mathrm{~g}$ & $5.10 \pm 0.63 \mathrm{~g}$ & $5.05 \pm 0.61 \mathrm{~g}$ \\
$\mathrm{IT}$ & $\mathrm{E} 2$ & $24.8 \pm 0.88 \mathrm{~g}$ & $5.10 \pm 0.56 \mathrm{~g}$ & $1.98 \pm 0.36 \mathrm{~g}$ \\
& $\mathrm{G} 1$ & $25.9 \pm 0.78 \mathrm{~g}$ & $4.80 \pm 0.86 \mathrm{~g}$ & $2.56 \pm 0.33 \mathrm{~g}$ \\
& $\mathrm{E} 2+\mathrm{G} 15$ & $24.8 \pm 0.78 \mathrm{~g}$ & $4.90 \pm 0.41 \mathrm{~g}$ & $4.48 \pm 0.55 \mathrm{~g}$ \\
\hline Total & & $25.2 \pm 0.49 \mathrm{~g}$ & $4.90 \pm 0.20 \mathrm{~g}$ & \\
\hline
\end{tabular}


Figure 1. (A) The PWT of rats before and $24 \mathrm{~h}$ after the incisional surgery. The PWT of the rats dropped significantly $24 \mathrm{~h}$ after the incisional surgery $* p<0.05$; (B) The PWT of rats before and after the incisional surgery of the intravenously (IV) group before administration of the indicated drug/drugs [17- $\beta$-estradiol (E2), GPER-selective agonist (G1), $\mathrm{E} 2+$ GPER-selective antagonist (G15)]. The PWT is presented as mean \pm standard error of the mean (SEM). There was no statistically significant difference between the groups; and (C) The PWT of rats before and after the incisional surgery of the intrathecal (IT) group before administration of the indicated drug/drugs (E2, G1, E2 + G15). The PWT was presented as mean \pm SEM in the figure. There was no statistically significant difference between the groups.

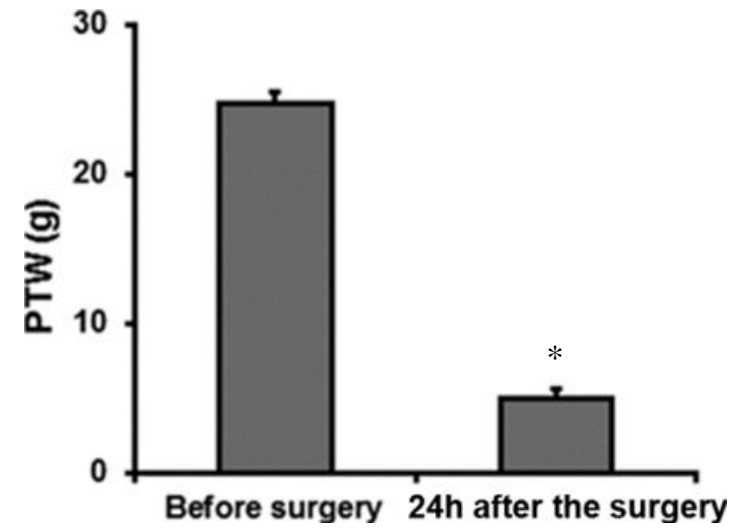

(A)

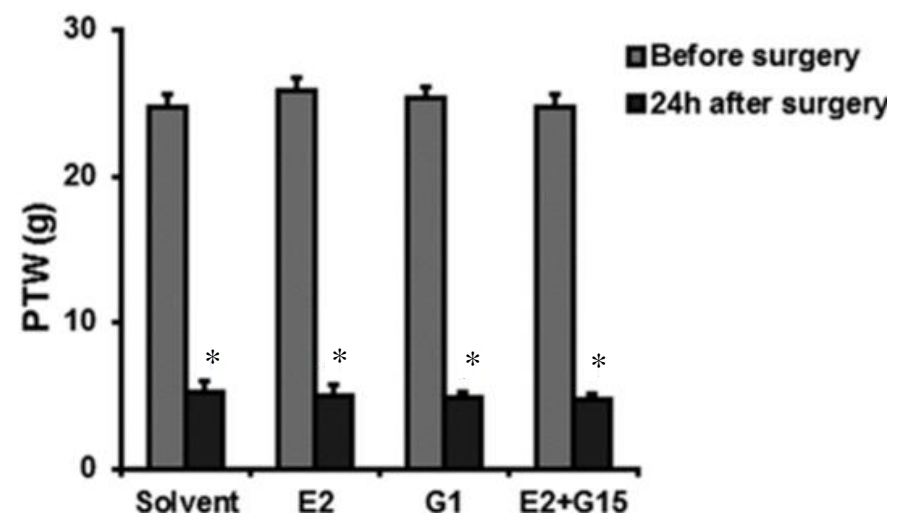

(B)

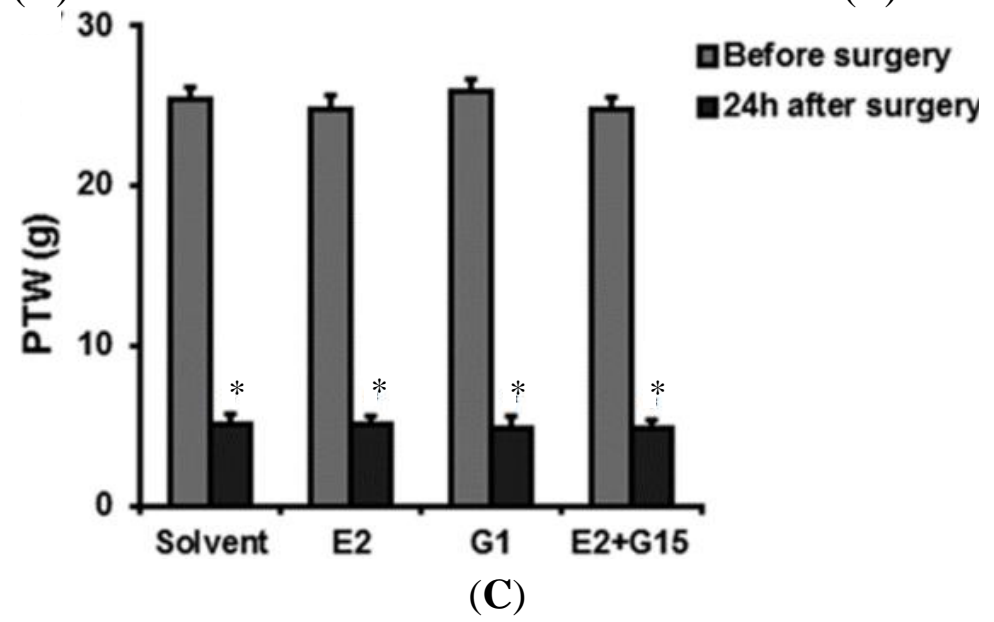

\subsection{Intravenously (IV) Group}

\subsubsection{The Effect of 17- $\beta$-Estradiol (E2) Administration on PWT}

Twenty-four hours after incisional surgery, a high dose of E2 was administered to the OVX rats through the caudal vein. The results showed a significant decrease in the PWT of the incisioned hind-paw within 30 min after the administration of the E2 compared with the solvent group (Table 1 and Figure 2A). 
Figure 2. (A) The PWT of rats before and $30 \mathrm{~min}$ after the administration of solvent and E2 through the caudal vein. The PWT dropped significantly $30 \mathrm{~min}$ after administration of E2. $* p<0.05$, compared with PWT before the administration; \# $p<0.05$, compared with the PWT of the solvent group after the drug administration; (B) The PWT of rats before and 30 min after the administration of solvent and G1 through the caudal vein. The PWT dropped significantly $30 \mathrm{~min}$ after $\mathrm{G}$ protein-coupled estrogen receptor (GPER)-selective agonist (G1) administration. * $p<0.05$, compared with the PWT before the administration; $\# p<0.05$, compared with the PWT of the solvent group after the drug administration; and (C) The PWT of rats before and $30 \mathrm{~min}$ after the administration of solvent and G15 + E2 through the caudal vein. The PWT of the G15 + E2 group decreased but this was not statistically significant.

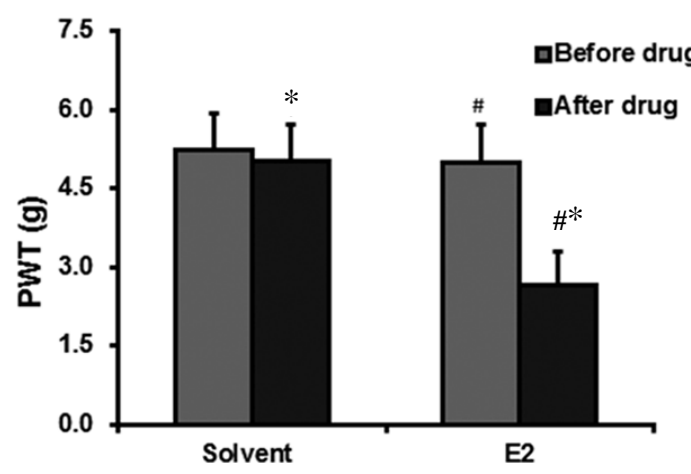

(A)

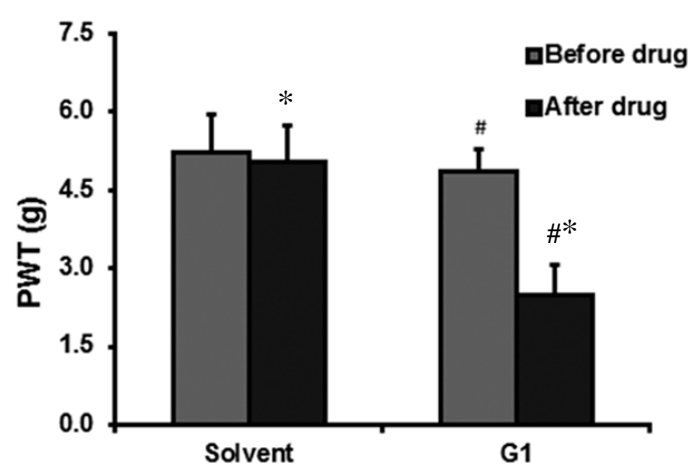

(B)

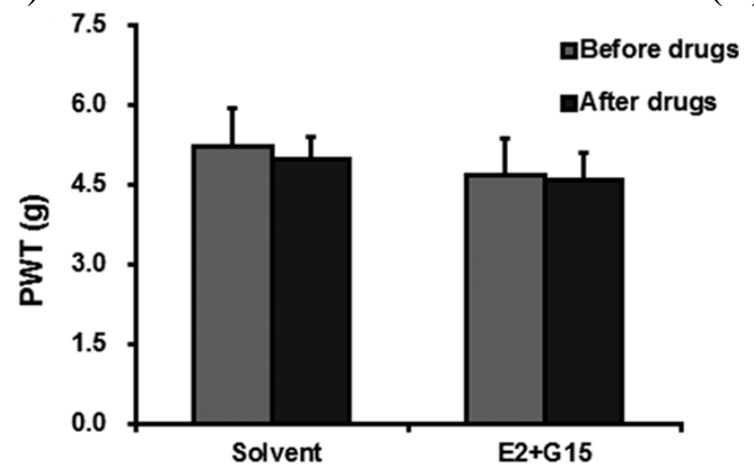

(C)

\subsubsection{G Protein-Coupled Estrogen Receptor (GPER)-Selective Agonist (G1) Administration}

\section{Ecreases PWT}

In order to investigate the hypothesis that GPER was involved in the rapid action of estrogen, the GPER-selective agonist G1 was administered. A single dose of G1 (3 $\mu \mathrm{g})$ was administered to OVX rats in the same way as E2. There was a significant difference between the PWTs of the pre-injection and post-injection group $(4.87 \pm 0.40$ and $2.50 \pm 0.58 \mathrm{~g}$, respectively; Figure $2 \mathrm{~B}$, $p<0.05 ; n=6)$.

\subsubsection{The Effect of 17- $\beta$-Estradiol (E2) + GPER-Selective Antagonist (G15) Administration on PWT}

To substantiate the finding that the estrogen receptor (ER) GPER mediates the above rapid effect of estrogen on pain modulation, whether G15, a GPER-selective antagonist, could block the effect of E2 
was investigated. Three minutes after the administration of E2, a single dose of G15 (E2:G15 = 1:7.4) was administered to the rats via the caudal vein. There was no significant difference between before drug administration and 30 min after the administration of E2 + G15 (Table 1 and Figure 2C).

\subsection{Intrathecal (IT) Group}

\subsubsection{The Effect of E2 Administration on PWT}

Twenty-four hours after incisional surgery, OVX rats were administered with E2 through the intrathecal catheter. The PWT around the wound decreased signficiantly from $5.10 \pm 0.56$ to $1.98 \pm 0.36 \mathrm{~g}$ ( $p=0.009$ ) within $20 \mathrm{~min}$. Administration of the solvent decreased the PWT from $5.10 \pm 0.63$ to $5.05 \pm 0.61 \mathrm{~g}$. The results showed that there was a significant decrease in the PWT of the incisioned hind-paw within 20 min after the administration of the E2 compared with the solvent group (Figure $3 \mathrm{~A}$ ).

Figure 3. (A) The PWT of rats before and $20 \mathrm{~min}$ after the administration of solvent and E2 through intrathecal catheter. The PWT dropped significantly $30 \mathrm{~min}$ after E2 adminisctration. $* p<0.05$, compared with the PWT before the administration; \# $p<0.05$, compared with the PWT of the solvent group after drug administration; (B) The PWT of rats before and $20 \mathrm{~min}$ after the administration of solvent and G1 through intrathecal catheter. The PWT dropped significantly $30 \mathrm{~min}$ after applied with G1. ${ }^{*} p<0.05$, compared with the PWT before the administration; \# $p<0.05$, compared with the PWT of the solvent group after the drug administration; and $(\mathbf{C})$ The PWT of rats before and 30 min after the administration of solvent and G15 + E2 through the intrathecal catheter.

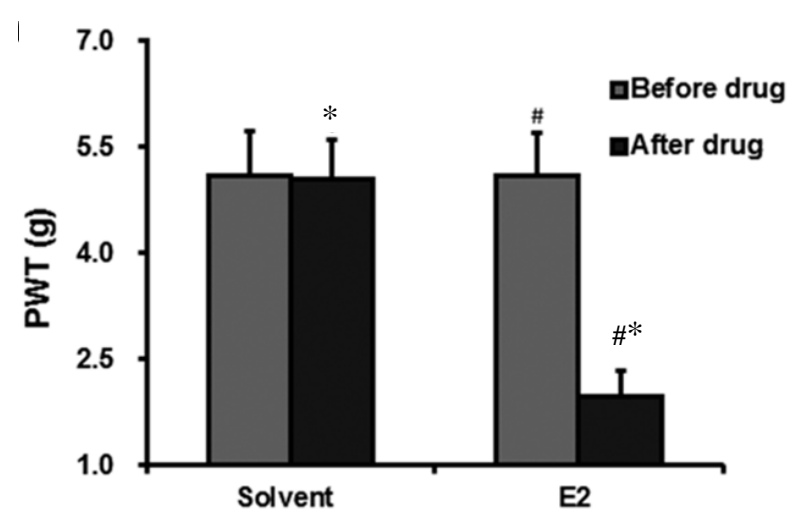

(A)

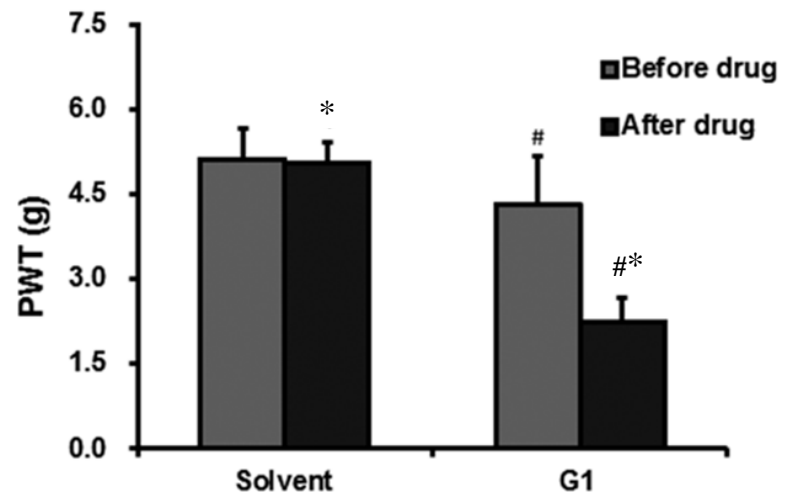

(B)

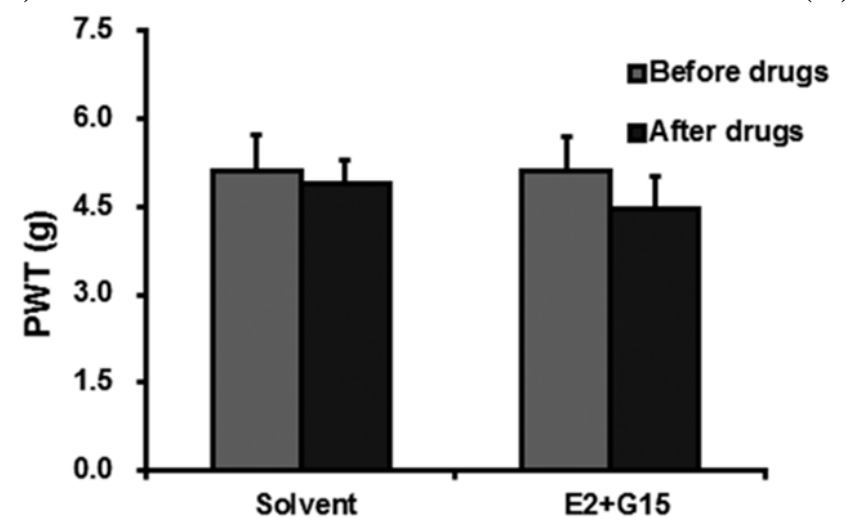

(C) 


\subsubsection{G1 Administration Decreases PWT}

Twenty-four hours after the incisional surgery, OVX rats were administered G1 through the intrathecal catheter. The PWT around the wound decreased significantly from $4.30 \pm 0.88$ to $2.25 \pm 0.42 \mathrm{~g}$ within $20 \mathrm{~min}$ (Table 1 and Figure 3B).

\subsubsection{The Effect of E2 + G15 Administration on PWT}

Twenty-four hours after incisional surgery, OVX rats were administered with G15 + E2 through the intrathecal catheter. The PWT around the wound decreased, however this decrease was not significant. There was no significant difference between the E2 + G15 group and the solvent group (Table 1 and Figure 3C).

\subsection{Discussion}

The present study aimed to identify the molecular signaling pathways involved in the estrogen-related regulation of pain. Previous studies have shown that estrogen can influence pain behavior in both humans and animals [19]. The PWT in animals is significantly reduced following subcutaneous injection of estrogen into the animal paw [9]. In accordance with previous research, the present study showed that administration of estrogen induced rapid mechanical hyperalgesia in OVX rats following incisional surgery.

The pathological effects of estrogen are mediated by several molecular signaling pathways, the primary pathway being activation of the estrogen receptors, namely ER $\alpha$ and ER $\beta$. These receptors are primarily located in the nucleus, belong to the steroid hormone receptor super-family and function as ligand-activated transcription factors. Estrogen regulates transcription through the ERs in two ways: (i) the classic activation whereby estrogen binds to the ERs, inducing transcription; and (ii) via the activator protein 1 (AP-1) enhancer elements pathway. The effect of estrogen via activation of the ERs by these pathways can often take hours if not days to be observed [24]. Interestingly, the present study reports a novel molecular signaling pathway of estrogen-related regulation of pain.

The $\mathrm{G}$ protein coupled estrogen receptors, GPERs, another form of estrogen receptors, are primarily located in the cell membrane. Estrogen can bind to GPERs acting rapidly through a nongenetic pathway. The GPERs were first isolated from B cells in 1996 by Carmeci et al. [25], and named GPR30 in 1997. The International Union of Pharmacology then officially named the receptor GPER. As a member of the G protein coupled receptor (GPCR) family, GPER has a 7-transmembrane hydrophobic domain, which consists of 20-26 amino acids interconnected by six alternating extracellular and intracellular loops [26]. Similar to GPCRs, GPER has an exceptionally conserved sequence, the Asp-Arg-Tyr triplet (DRY). DRY is located in the second intra-cytoplasmic loop after the third transmembrane domain, and is believed to play a role in signal transduction [27]. Several studies have shown that E2 can induce a rapid increase (within minutes and even seconds) of intracellular cAMP and $\mathrm{Ca}^{2+}$ concentration through GPER [1,15-17]. It has been reported both in vitro and in vivo that $\mathrm{E} 2$ can act rapidly, and it has been speculated that the receptor involved in this rapid effect of E2 was GPER [7,9,19]. Consequently, in the present study, the GPER's selective agonists G1 and antagonist G15 were investigated. 
The GPER-specific compound 1, G1, a substituted tetrahydro-3H-cyclopenta[c]-quinoline, does neither bind nor activate the classical nuclear receptors, but rather binds to GPER with high affinity and selectivity [28]. A G1 analog, G15, binds to GPER with high affinity, however has no activating effects. G15 does not bind to either ER $\alpha$ or ER $\beta$. It has been shown in vivo that G15 can block some of estrogen's uterine and neurological responses and that GPER contributes to the effects initiated by estrogen [17]. The results of the current study are in agreement with these findings, since administration of G1 or G15 + E2 decreased the PWT. suggesting that estrogen rapidly enhanced the incisional pain of OVX rats primarily through the GPER.

GPER in the spinal cord may mediate the rapid effects of estrogen.

When E2 and G1 were administered intrathecally, the effects on PWT were more rapid and more significant than intravenous, indicating that E2 may act through activating the GPER in the spinal cord. There are many factors in pain progression, and each step can influence pain [14]. The unmyelinated $\mathrm{C}$ fibre, the cell body located in the dorsal root ganglia, primarily responds to mechanical, thermal and chemical stimulation [29]. In rats, GPER mRNA is expressed in the spinal cord, dorsal root ganglia, no dose ganglia, trigeminal ganglia, hippocampus, brain stem and hypothalam0us [30]. In the spinal cord, GPER is detected in the dorsal horn, particularly in the superficial layer [30,31]. Interestingly, aromatase (estrogen-synthase, which catalyzes the conversion of C19 androgens into estrogens) is also expressed specifically in the superficial layer of the dorsal horn [24].

To date, few studies have investigated the mechanisms for the regulation of GPER expression. It has been shown however, that GPER expression is down-regulated in DRG neurons of OVX female rats, and that the reduction in GPER expression can be recovered by estrogen replacement [31].

The current study consists of several limitations including the minimal evaluation of pain. A combination of pain evaluation methods would be beneficial, more accurate, and might further support the results. Only one dose of each drug was investigated. A test-dose response curve would add further insight into the findings of the study. Finally, further molecular biology investigations would provide a possible molecular mechanism for analgesic drugs.

\section{Materials and Methods}

\subsection{Animals}

Behavioral experiments were performed on female Sprague-Dawley (SD) rats (100-150 g). Animals were obtained from the Shanghai Experimental Animal Center (Chinese Academy of Sciences, Shanghai, China) and housed in a controlled environment in a $12 \mathrm{~h}$ light: $12 \mathrm{~h}$ dark cycle. Food and water were available ad libitum. Female rats underwent ovariectomy (OVX) 2 days after being housed at the lab. The study was conducted in accordance with applicable guidelines for animal research and was approved by the Ethical and Research Committees of Shanghai First People's Hospital (2009, No. 03). 


\subsection{Drugs}

17- $\beta$-Estradiol (E2) was purchased from Sigma, Hong Kong, China. G1 and G15 were purchased from Tocris, Shanghai, China. All drugs were dissolved in sterilized phosphate buffered saline (PBS) solvent and $10 \%$ dimethyl sulfoxide.

\subsection{Equipment}

The VonFrey filaments were purchased from Stolting, Wood Dale, IL, USA (0.008-300 g). The intrathecal catheter [PE-10 (PE-0503)] tube, consisted of an outer diameter of $0.5 \mathrm{~mm}$ and an inner diameter of $0.25 \mathrm{~mm}$ (AniLab Software \& Instruments Co., Ltd., Ningbo, China). The catheter was cut into $10 \mathrm{~cm}$, a mark made at $7.5 \mathrm{~cm}$ and sterilized by ethylene oxide. The IT injection was operated using a $20 \mu \mathrm{L}$-micro-syringe (Shanghai Medical Instruments Co., Ltd., Shanghai, China).

\subsection{Ovariectomized (OVX) Surgery}

Rats were anesthetized by inhalation of $3 \%$ isoflurane with pure oxygen. A $2-3 \mathrm{~cm}$ longitudinal incision was made using an aseptic technique. The incision was then pulled laterally to open the peritoneum, white adipose tissue observed, the ovaries and fallopian tubes identified, and the ovaries removed. The skin was sutured and penicillin administered to prevent infection. The rats were housed separately following surgery.

\subsection{The Incisional Pain Model}

Between 14 and 20 days after ovariectomy, the incisional pain model was performed as previously described [32]. A $1 \mathrm{~cm}$ longitudinal incision was made through the skin, fascia and muscle of the plantar aspect of the left hind-paw under $2 \%$ isoflurane anesthesia. The skin was then sutured with two mattress sutures of 4-0 nylon. After surgery, the animals were housed individually.

\subsection{Subarachnoid Catheter}

Subarachnoid catheter placement was performed as previously described [32]. Rats were anesthetized by inhalation of 3\% isoflurane with pure oxygen, and placed in a special device for intrathecal (IT) catheterization. The fur on the back of the rats was shaved, confirmation of the depth of anesthesia noted and breathing monitored. A $3 \mathrm{~cm}$ incision along the midpoints of the ears was made. Subcutaneous fat and superficial fascia were removed to expose the neck muscles. The neck muscles were isolated along the midline and the foramen magnum exposed. The dura mater was carefully pricked and the cerebrospinal fluid expelled. The PE-10 catheter was carefully placed into the subarachnoid space approximately $7.5 \mathrm{~cm}$ through the foramen magnum. Position of the catheter, sutured muscles and skin were confirmed. Indwelling 1-2 cm catheter in the neck was fixed to the skin of the neck, and burnt at the end of the catheter to close it. The rats recovered in individual cages after surgery. Three days after successful placement of the catheter, the surgical rats were ready for the incisional model. 


\subsection{Evaluation of the Incisional Pain Threshold}

The mechanical threshold, determined as the hind-paw withdrawal threshold (PWT) was measured using von Frey filaments as described previously [33]. Briefly, rats were placed into individual containers and acclimatized to the test chambers for $15 \mathrm{~min}$. A series of nine von Frey filaments $(0.4,0.7,1.2,2.0,3.6,5.5,8.5,15$ and $26 \mathrm{~g})$ were applied vertically to the plantar surface of the hind-paw for $7 \mathrm{~s}$ while the filament was bent. Positive responses included an abrupt withdrawal of the hind-paw from the stimulus or flinching behavior immediately following removal of the stimulus. In the absence of a response at a pressure of $26 \mathrm{~g}$, a cut-off value was assigned to the animal. Tests were performed in duplicate with an approximate 3 min resting period between withdrawal responses. The estimate of mechanical pain threshold (EI50) was calculated by the Equation (1):

$$
\mathrm{EI} 50=X_{\mathrm{f}}+k \times d
$$

where $X_{\mathrm{f}}$ is the final test level, $d$ is the log interval between intensities and $k$ is the tabulated value for maximum likelihood estimate. The PWT of the two hind-paws of the rats were tested before the incisional surgery, $24 \mathrm{~h}$ afterward surgery and $30 \mathrm{~min}$ after the administration of drugs.

\subsection{Administration of Drugs}

Drug administration was performed $24 \mathrm{~h}$ after the incisional surgery in order to allow assessment of the action of analgesics on an established pain state and to remove the possibility that pain-relieving treatment during surgery or in the immediate postsurgical period could not alter the development of the behavioral hypersensitivity [34].

Twenty-four hours after the incisional operation, all the rats were coded, and randomly divided into two groups. In the intravenously (IV) group, the drugs were administered through the caudal vein of the rat, in the IT group drugs were administered through intrathecal catheter. Each group was divided into four subgroups: A, the E2 group was administered E2; B, the G1 group, was administered G1; C, the G15 group, was administered E2 + G15; and D, the control group, was administered the same volume of solvent. The researcher who performed the von Frey test was blind to the drugs given to the rats and the grouping of the rats.

\subsection{Doses of the Drugs}

IV group: The dose of E2 was $1000 \mathrm{ng}, \mathrm{G} 13000 \mathrm{ng}, \mathrm{G} 15+\mathrm{E} 2$ of 10,000 + $1000 \mathrm{ng}$ with G15 administered $3 \mathrm{~min}$ after E2 administration. IT group: The dose of E2 was $10 \mathrm{ng}, \mathrm{G} 110 \mathrm{ng}$, G15 + E2 of $10+10 \mathrm{ng}$ with G15 and E2 premixed before administration [6,9,10,19,20,24].

\subsection{Statistical Analysis}

The main data presented are the mechanical thresholds (PWT) of the rats at different periods. All data are reported as mean \pm standard error of the mean (SEM). All statistical comparisons were made with one-way analysis of variances (ANOVAs) followed by post hoc comparisons using SPSS 18.0, IBM, Armonk, NY, USA. A $p$ value of $p<0.05$ was considered statistically significant. 


\section{Conclusions}

Estrogen can rapidly regulate the incisional pain of OVX rats, whether administered through the caudal vein or the intrathecal catheter. The regulation of pain via E2 is modulated through the $\mathrm{G}$ protein-coupled estrogen receptor, primarily, spinal cord GPER.

\section{Acknowledgments}

We thank Junyan Yao from Shanghai First People's Hospital for outstanding advices.

\section{Author Contributions}

Guanghui An performed the analysis and interpretation of data, and drafted the manuscript. Wenhui Li participated in acquisition of data. Tao Yan revised the manuscript for important intellectual content. Shitong Li participated in the study concept and design. All authors read and approved the final manuscript.

\section{Conflicts of Interest}

The authors declare no conflict of interest.

\section{References}

1. Craft, R.M. Modulation of pain by estrogens. Pain 2007, 132 (Suppl. 1), S3-S12.

2. Chesterton, L.S.; Barlas, P.; Foster, N.E.; Baxter, G.D.; Wright, C.C. Gender differences in pressure pain threshold in healthy humans. Pain 2003, 101, 259-266.

3. Sarlani, E.; Farooq, N.; Greenspan, J.D. Gender and laterality differences in thermosensation throughout the perceptible range. Pain 2003, 106, 9-18.

4. Frot, M.; Feine, J.S.; Bushnell, M.C. Sex differences in pain perception and anxiety. A psychophysical study with topical capsaicin. Pain 2004, 108, 230-236.

5. Unruh, A.M. Gender variations in clinical pain experience. Pain 1996, 65, 123-167.

6. Khasar, S.G.; Dina, O.A.; Green, P.G.; Levine, J.D. Estrogen regulates adrenal medullary function producing sexual dimorphism in nociceptive threshold and beta-adrenergic receptor-mediated hyperalgesia in the rat. Eur. J. Neurosci. 2005, 21, 3379-3386.

7. Hucho, T.; Levine, J.D. Signaling pathways in sensitization: Toward a nociceptor cell biology. Neuron 2007, 55, 365-376.

8. Woolf, C.J.; Ma, Q. Nociceptors-Noxious stimulus detectors. Neuron 2007, 55, 353-364.

9. Hucho, T.B.; Dina, O.A.; Kuhn, J.; Levine, J.D. Estrogen controls PKCe-dependent mechanical hyperalgesia through direct action on nociceptive neurons. Eur. J. Neurosci. 2006, 24, 527-534.

10. Evrard, H.C.; Balthazart, J. Rapid regulation of pain by estrogens synthesized in spinal dorsal horn neurons. J. Neurosci. 2004, 24, 7225-7229.

11. Mannino, C.A.; South, S.M.; Quinones-Jenab, V.; Inturrisi, C.E. Estradiol replacement in ovariectomized rats is antihyperalgesic in the formalin test. J. Pain 2007, 8, 334-342. 
12. Gaumond, I.; Arsenault, P.; Marchand, S. Specificity of female and male sex hormones on excitatory and inhibitory phases of formalin-induced nociceptive responses. Brain Res. 2005, 1052, 105-111.

13. Ceccarelli, I.; Scaramuzzino, A.; Massafra, C.; Aloisi, A.M. The behavioral and neuronal effects induced by repetitive nociceptive stimulation are affected by gonadal hormones in male rats. Pain 2003, 104, 35-47.

14. Sanoja, R.; Cervero, F. Estrogen modulation of ovariectomy-induced hyperalgesia in adult mice. Eur. J. Pain 2008, 12, 573-581.

15. Revankar, C.M.; Cimino, D.F.; Sklar, L.A.; Arterburn, J.B.; Prossnitz, E.R. A transmembrane intracellular estrogen receptor mediates rapid cell signaling. Science 2005, 307, 1625-1630.

16. Bjornstrom, L.; Sjoberg, M. Mechanisms of estrogen receptor signaling: Convergence of genomic and nongenomic actions on target genes. Mol. Endocrinol. 2005, 19, 833-842.

17. Dennis, M.K.; Burai, R.; Ramesh, C.; Petrie, W.K.; Alcon, S.N.; Nayak, T.K.; Bologa, C.G.; Leitao, A.; Brailoiu, E.; Deliu, E.; et al. In vivo effects of a GPR30 antagonist. Nat. Chem. Biol. 2009, 5, 421-427.

18. Khasar, S.G.; Lin, Y.H.; Martin, A.; Dadgar, J.; McMahon, T.; Wang, D.; Hundle, B.; Aley, K.O.; Isenberg, W.; McCarter, G.; et al. A novel nociceptor signaling pathway revealed in protein kinase C epsilon mutant mice. Neuron 1999, 24, 253-260.

19. Kuhn, J.; Dina, O.A.; Goswami, C.; Suckow, V.; Levine, J.D.; Hucho, T. GPR30 estrogen receptor agonists induce mechanical hyperalgesia in the rat. Eur. J. Neurosci. 2008, 27, 1700-1709.

20. Tang, B.; Ji, Y.; Traub, R.J. Estrogen alters spinal NMDA receptor activity via a PKA signaling pathway in a visceral pain model in the rat. Pain 2008, 137, 540-549.

21. Dai, Y.; Fukuoka, T.; Wang, H.; Yamanaka, H.; Obata, K.; Tokunaga, A.; Noguchi, K. Contribution of sensitized P2X receptors in inflamed tissue to the mechanical hypersensitivity revealed by phosphorylated ERK in DRG neurons. Pain 2004, 108, 258-266.

22. Ma, B.; Yu, L.H.; Fan, J.; Cong, B.; He, P.; Ni, X.; Burnstock, G. Estrogen modulation of peripheral pain signal transduction: Involvement of P2X3 receptors. Purinergic Signal. 2011, 7, 73-83.

23. Fan, J.; Yu, L.H.; Zhang, Y.; Ni, X.; Ma, B.; Burnstock, G. Estrogen altered visceromotor reflex and P2X3 mRNA expression in a rat model of colitis. Steroids 2009, 74, 956-962.

24. Evrard, H.C. Estrogen synthesis in the spinal dorsal horn: A new central mechanism for the hormonal regulation of pain. Am. J. Physiol. Regul. Integr. Comp. Physiol. 2006, 291, R291-R299.

25. Carmeci, C.; Thompson, D.A.; Ring, H.Z.; Francke, U.; Weigel, R.J. Identification of a gene (GPR30) with homology to the G-protein-coupled receptor superfamily associated with estrogen receptor expression in breast cancer. Genomics 1997, 45, 607-617.

26. Prossnitz, E.R.; Arterburn, J.B.; Smith, H.O.; Oprea, T.I.; Sklar, L.A.; Hathaway, H.J. Estrogen signaling through the transmembrane $\mathrm{G}$ protein-coupled receptor GPR30. Annu. Rev. Physiol. 2008, 70, 165-190.

27. Strader, C.D.; Fong, T.M.; Tota, M.R.; Underwood, D.; Dixon, R.A. Structure and function of G protein-coupled receptors. Annu. Rev. Biochem. 1994, 63, 101-132. 
28. Bologa, C.G.; Revankar, C.M.; Young, S.M.; Edwards, B.S.; Arterburn, J.B.; Kiselyov, A.S.; Parker, M.A.; Tkachenko, S.E.; Savchuck, N.P.; Sklar, L.A.; et al. Virtual and biomolecular screening converge on a selective agonist for GPR30. Nat. Chem. Biol. 2006, 2, 207-212.

29. Julius, D.; Basbaum, A.I. Molecular mechanisms of nociception. Nature 2001, 413, 203-210.

30. Dun, S.L.; Brailoiu, G.C.; Gao, X.; Brailoiu, E.; Arterburn, J.B.; Prossnitz, E.R.; Oprea, T.I.; Dun, N.J. Expression of estrogen receptor GPR30 in the rat spinal cord and in autonomic and sensory ganglia. J. Neurosci. Res. 2009, 87, 1610-1619.

31. Takanami, K.; Sakamoto, H.; Matsuda, K.; Hosokawa, K.; Nishi, M.; Prossnitz, E.R.; Kawata, M. Expression of $\mathrm{G}$ protein-coupled receptor 30 in the spinal somatosensory system. Brain Res. 2010, 1310, 17-28.

32. Brennan, T.J.; Vandermeulen, E.P.; Gebhart, G.F. Characterization of a rat model of incisional pain. Pain 1996, 64, 493-501.

33. Chaplan, S.R.; Bach, F.W.; Pogrel, J.W.; Chung, J.M.; Yaksh, T.L. Quantitative assessment of tactile allodynia in the rat paw. J. Neurosci. Methods 1994, 53, 55-63.

34. Valenzano, K.J.; Tafesse, L.; Lee, G.; Harrison, J.E.; Boulet, J.M.; Gottshall, S.L.; Mark, L.; Pearson, M.S.; Miller, W.; Shan, S.; et al. Pharmacological and pharmacokinetic characterization of the cannabinoid receptor 2 agonist, GW405833, utilizing rodent models of acute and chronic pain, anxiety, ataxia and catalepsy. Neuropharmacology 2005, 48, 658-672.

(C) 2014 by the authors; licensee MDPI, Basel, Switzerland. This article is an open access article distributed under the terms and conditions of the Creative Commons Attribution license (http://creativecommons.org/licenses/by/3.0/). 